\title{
Robot-assisted radical prostatectomy: Another Canadian experience
}

\author{
Roger Valdivieso MD; ${ }^{*}$ Kevin C. Zorn, MD, FACS, FRCSC ${ }^{\dagger}$ \\ *University of Montreal Hospital Center (CHUM); 'Director of Robotic Urological Surgery - CHUM, Assistant Professor, University of Montreal Hospital Center (CHUM)
}

See related article on page 92.

Cite as: Can Urol Assoc J 2014;8(3-4):98-99. http://dx.doi.org/10.5489/cuaj.2083 Published online April 14, 2014.

$\mathrm{O}$ ver the past decade, robot-assisted radical prostatectomy (RARP) has been gaining acceptance among patients and urologists. It has become the dominant surgical approach in the United States and other countries. Despite a paucity of prospective, randomized trials supporting its oncological or functional superiority over open radical prostatectomy (ORP), ${ }^{1}$ parameters of reduced blood loss and transfusion, shorter hospital stay, reduced re-admission, re-operation, ureteral and rectal injuries, lymphocele and anastomotic leak, as well as overall reduced surgical morbidity, have favoured adoption of RARP. ${ }^{2}$

In the current Canadian study, the authors present their retrospective, single institution review of perioperative, oncological and functional outcomes for both ORP and RARP. ${ }^{3,4}$ Interestingly at this tertiary academic high volume centre (The Vancouver Prostate Centre BC), their findings contrast to previously published outcomes. ${ }^{5}$ More specifically, systemic reviews suggest that RARP is advantageous with regards to blood loss, transfusion rates and length of hospital stay. In the study by Gagnon and colleagues, ORP and RARP blood loss (402 vs. $287 \mathrm{~mL})$, transfusion rate $(1.5 \%$ vs. $3.5 \%)$ and length of stay (1.95 vs. 1.69 days) were not found to be statistically significant (all $p>0.05$ ). ${ }^{3}$ Furthermore, no differences in 90-day non-descriptive Clavien complications were observed. Moreover, in our initial robotic experience at the University of Montreal Hospital Centre in 2012, we compared perioperative outcomes of the first 200 RARP cases to 83 ORP cases performed in the same year (unpublished data). RARP men demonstrated significantly less blood loss (625 vs. $237 \mathrm{~mL}$; $p<0.01)$, transfusion $(16.5 \%$ vs. $1.8 \% ; p<0.01)$ and length of stay (3.1 days vs. 1.4 days; $p<0.01$ ). The incidence of perioperative 30-day complications was also higher in the ORP group $(39 \%$ vs. $9 \%, p<0.01)$. Similarly, in a large meta-analysis by Tewari and colleagues ${ }^{2}$ of 167184 ORP and 62309 RARP cases from 400 original publications, the mean blood loss (745 vs. $188 \mathrm{~mL}, p<0.01)$, transfusion rate $(16.5 \%$ vs. $1.8 \%, p<0.01)$ and length of stay (3.1 vs. $1.4 \mathrm{~d}, p<0.01$ ) were all more favourable in RARP. In addition, rates for re-admission, re-operation, nerve, ureteral and rectal injury, deep vein thrombosis, pneumonia, hematoma, lymphocele, anastomotic leak, fistula, and wound infection showed significant differences favouring RARP over ORP.

Two factors that may explain the discrepancy are surgeon experience and learning curve. A single surgeon (SLG), who did not undergo formal robotic fellowship training, performed the 200 consecutive RARP cases. It is noteworthy to highlight that only 70 cases were conducted before the study period. As such, the oncological learning curve of 200 $(n=4)$ cases was not reached for this surgeon and therefore, the comparison to a high-volume expert ORP surgeon could be considered unfair. More specifically, the reader should be aware that the single ORP surgeon (MEG) was fellowshiptrained at the MD Anderson Center and has an incredible experience of over 2500 ORP cases. Regardless of the lack of RARP expertise, what is meaningful from this study is that for an open surgeon who converts to the robotic approach there appears to be no harm in the early experience.

The similarity of oncological parameters and functional outcomes at 6 and 12 months, were also observed. Ideally, 1 - and 3-month continence outcomes (strict pad-free definition) should have also been reported as earlier recovery has been demonstrated in several reports..$^{2,6}$ Interestingly, pT2-PSM rates (22\% vs. $17 \%$ ) appear moderately high when compared to high volume surgeons $(4 \%-16 \%))^{2,7}$ Similarly, there is a concern for the lymph node yield for both ORP (5.4) and RARP (3.9) groups when compared to contemporary reported series, ${ }^{8}$ particularly for high-risk patients where 
extended pelvic lymph node dissection is recommended. This likely suggests that a limited template was used during this study. Nevertheless, their results further provide evidence that men undergoing RARP are not disadvantaged in terms of oncological outcomes.

Moreover, the authors briefly describe ORP modifications based on the technique already described by Walsh and colleagues. ${ }^{9}$ Acharya and colleagues have previously reported on the positive impact of minimally invasive techniques on ORP practice. ${ }^{10}$ Such adaptations include a shorter incision, avoidance of surgical drains for "watertight" anastamosis, incisional injection of anaesthesia and use of systemic ketorolac aiming an earlier discharge at 1 day postoperation. Criteria for discharge, particularly for hemoglobin levels, were unfortunately not mentioned by the authors. Their changes yielded outstanding perioperative results that are worthy of publication. These would be meaningful to other community urologists in practice to potentially deliver improved ORP patient care and help reduce costs.

Despite global acceptance, RARP is not widely disseminated in current Canadian urological practice. Consequently, very few Canadian centres have reported their experience with the daVinci system (Intuitive Surgical, Inc.). This study is meaningful in that it not only adds to the Canadian RARP experience, it also clearly demonstrates that even a welltrained, laparoscopic-naive surgeon in his/her early learning curve can accomplish outcomes at least as good as an expert ORP surgeon. It will be of great interest to the urological community to see the same group analysis assessing the latest RARP caseload (beyond the learning curve) to see if such outcomes, including overall cost, remain comparable.
Competing interests: Dr. Valdivieso declares no competing financial or personal interests. Dr. Zorn is involved with AMS in GreenLight training.

\section{References}

1. Montorsi F, Wilson TG, Rosen RC, et al. Best practices in robot-assisted radical prostatectomy: recommendations of the Pasadena Consensus Panel. Eur Urol 2012;62:368-81.

2. Tewari $A$, Sooriakumaran $P$, Bloch $D A$, et al. Positive surgical margin and perioperative complication rates of primary surgical treatments for prostate cancer: A systematic review and meta-analysis comparing retropubic, laparoscopic, and robotic prostatectomy. Eur Urol 2012;62:1-15.

3. Gagnon LO, Goldenberg SL, Lynch K, et al. Comparison of open and robotic-assisted prostatectomy: The University of British Columbia experience. Can Urol Assoc J 2014;8:92-7. http://dx.doi.org/10.5489/ cuaj. 1707

4. Pilecki MA, McGuire BB, Jain $\mathrm{U}$, et al. National multi-institutional comparison of 30-day postoperative complication and readmission rates between open retropubic radical prostatectomy and robot-assisted laparoscopic prostatectomy using NSQIP. J Endourol 2014;28:430-6. http://dx.doi.org/10.1089/ end.2013.0656. Epub 2014 Jan 4.

5. Novara G, Ficarra V, Mocellin S, et al. Systematic review and meta-analysis of studies reporting oncologic outcome after robot-assisted radical prostatectomy. Eur Urol 2012;62:382-404.

6. Secin FP, Savage C, Abbou C, et al. The learning curve for laparoscopic radical prostatectomy: an international multicenter study. J Urol 2010;184:2291-6.

7. Novara G, Ficarra V, Mocellin S, et al. Systematic review and meta-analysis of studies reporting oncologic outcome after robot-assisted radical prostatectomy. Eur Urol 2012;62:382-404.

8. Yuh B, Artibani W, Heidenreich A, et al. The role of robot-assisted radical prostatectomy and pelvic lymph node dissection in the management of high-risk prostate cancer: A systematic review. Eur Urol 2014;65:918-27.

9. Walsh PC. Radical and perineal prostatectomy In: A W, editor. Campbell-Walsh Urology. 10th edition. Philadelphia, PA: Elsevier; 2011.

10. Acharya SS, Zorn KC, Shikanov S, et al. Evolution of open radical retropubic prostatectomy-how have open surgeons responded to the challenge of minimally invasive surgery? J Endourol 2009;23:1893-7.

Correspondence: Dr. Kevin C. Zorn, Assistant Professor of Surgery, Minimally Invasive UrologicalOncologist, Director of Robotic Surgery, University of Montreal Hospital Centre, 235 Rene Levesque, Est Suite 301, Montreal, QC H2X 1N8 